\title{
A Multi-Fidelity Approach for Supporting Early Aircraft Design Decisions
}

\author{
John J Doherty $^{\mathrm{a}}{ }^{\text {, Stephen R H Dean }}{ }^{\mathrm{b}}$, Paul Ellsmore ${ }^{\mathrm{b}}$ and Andrew Eldridge ${ }^{\mathrm{b}}$ \\ ${ }^{\mathrm{a}}$ Technical Fellow, QinetiQ Ltd, Farnborough, UK. \\ ${ }^{\mathrm{b}}$ Aerospace Consultancy, QinetiQ Ltd, Farnborough, UK.
}

\begin{abstract}
The QinetiQ Aerospace Consultancy group has been actively developing and applying process automation and optimisation capabilities in support of air vehicle assessment and design for over 20 years. These capabilities have evolved greatly during this timeframe from their initial origins as research activities, into mature capabilities for underpinning decision making in both civil and military air vehicle projects. In parallel the same generic approaches have also found usage in weapons, maritime and motorsport design. In recent years effort has focussed on enhancing a number of different, but complementary, capabilities at QinetiQ, each of which have different advantages and disadvantages, but which together better address the needs of air vehicle assessment and design. These capabilities are linked by a common requirement to assess widely differing characteristics concurrently, in order to model the consequences of design decisions, such as technology and system choices, in terms of the overall impact on an air vehicle project. This paper describes these tools in the context of their use within the Integrated Wing project.
\end{abstract}

Keywords: Aircraft Concept, Design Space, Requirements Selection, Multi-disciplinary Design, Optimisation.

\section{Introduction}

Within the aerospace industry a typical air vehicle design project can be broadly described by a number of different phases: feasibility, conceptual design, preliminary design, detailed design and manufacturing design. The feasibility phase identifies the customer need and the manufacturer's potential top level business offering and hence establishes the overall business case for the air vehicle design. The conceptual design phase, supported by initial preliminary design activities, evolves potential air vehicle concepts and associated performance datasets, in order to down-select the final air vehicle concept. During the remaining phases the definition of this selected air vehicle concept is significantly refined in order to provide the target for subsequent production.

\footnotetext{
${ }^{1}$ Technical Fellow, Aerospace Consultancy, X80 Building, QinetiQ Ltd, Cody Technology Park, Ively Road, Farnborough, Hants, GU14 OLX, UK; E-mail: JJDoherty @ QinetiQ.com.
} 
It is widely recognized that this conventional aerospace design process is nonideal. In particular major decisions must frequently be made in each phase based upon information which is insufficiently detailed and immature. More detailed and mature information can potentially be generated using the analysis and design toolsets normally employed in subsequent phases, but generally this cannot be achieved within the timescales dictated by earlier phases and, in addition, often cannot be generated for a sufficient number of possible design alternatives. This issue is particularly constraining at the early stages of design when major design freedoms/decisions associated with the choice of the air vehicle concept and its associated technologies and systems are still open, but these must then be narrowed/down-selected without having access to all the desired supporting information. In order to reduce risk during the design process, the overall business case must be regularly re-evaluated as further information becomes available, in order to check the continued success of the overall project. Inevitably this information often comes too late to fundamentally rethink early design decisions and final compromise solutions may often result.

This conventional aerospace design process is facing even greater challenges for addressing the design of future air vehicles. Operational performance drivers for both military and civil air vehicles are becoming increasingly demanding, potentially leading to the future adoption of more novel air vehicles, employing novel technologies and systems. For military air vehicles the thrust is towards mission flexibility, improved survivability and use of unmanned air vehicles. Stringent environmental targets for civil aircraft, such as the ACARE 2020 Vision [1] for 50\% reduction in $\mathrm{CO} 2$ and $80 \%$ reduction in NOX, mean that novel aircraft configurations, employing novel technologies such as flow control and extensive use of composites, must be considered. The addition of these novel factors into the existing non-ideal design process means alternative approaches for supporting design decisions are required, in order to predict and hence decide upon the best combination of aircraft concept, technologies and systems.

\section{Integrated Wing Programme}

The Integrated Wing Aerospace Technology Validation Programme (IWATVP) [2] brings together industry and researchers within a UK national project funded jointly by the UK Department for Business, Enterprise \& Regulatory Reform (DBERR) and the industrial partners. The overall aim of the project is to validate technologies which can lead to a step change in performance for future aircraft, in order to address challenging future operational performance requirements best characterised by the ACARE 2020 Vision.

As part of the IWATVP project QinetiQ leads a Work Package focused on Requirements Integration and Optimisation (WPI) shown in Figure 1. A key objective for QinetiQ within WPI is to research and demonstrate the potential for alternative analysis and design approaches to support decision making in the early stages of design. Capabilities for process automation and optimisation, operating at different levels of modeling fidelity, are reasonably well established at QinetiQ and have been used for a number of years for other air vehicle design studies. Within 
WPI the potential for these alternative approaches will be demonstrated for supporting design decisions in the context of both conventional and novel civil aircraft concepts, encompassing associated conventional and novel technologies and system choices.

Two approaches for supporting design decisions are being investigated within WPI. The first addresses the selection of technologies and systems in the context of broad design space exploration. The second approach enables a more detailed investigation of the impact of technology and system choices for specific aircraft concept types using a multi-disciplinary design optimisation approach. These capabilities, together with a discussion of how they would be used to support the design process, are described in the remainder of this paper.

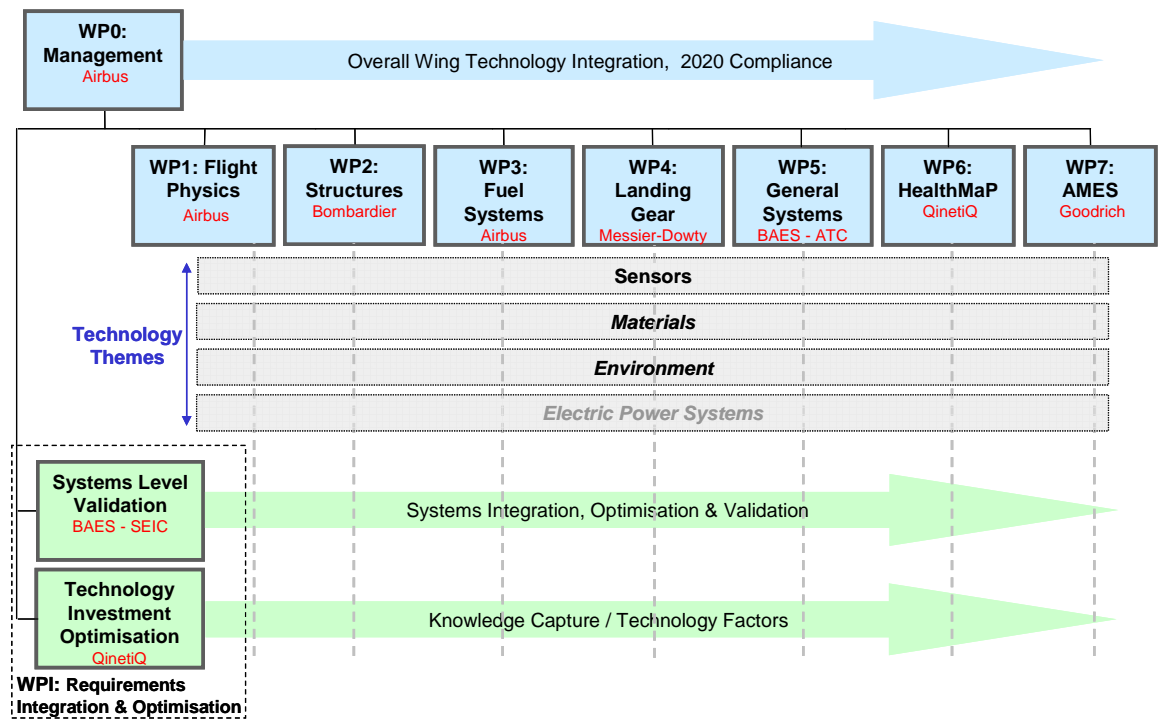

Figure 1. The Integrated Wing Programme Structure

\section{Multi-level Support for Design Decisions}

In the early stages of aircraft design, the traditional approach to assessing technology or system choices is through designing a baseline aircraft concept, followed by parametric modeling of the effect of different choices of technologies or systems, in order to assess the potential benefits, costs and risks associated with each design choice. Although this approach can provide a reasonable level of modeling fidelity and hence an understanding of the main trades and payoffs of design choices for the aircraft concept, it reduces the applicability of the assessment to a small area of the concept design space.

An alternative approach is to use a more generalized concept modeling approach, which requires less detail of specific geometry features, in order to avoid 
constraining the study to a specific region of the concept design space. In particular aircraft concepts are defined by parameters that primarily represent the desired performance characteristics, rather dictating specific geometry detail. By avoiding the need to down-select a specific aircraft concept (including assumptions about the mission and what level of technology is used), a study does not need to be constrained by existing concept assumptions or constraints that may limit the applicability of certain technologies. In particular it is very likely that certain technologies or systems will be more beneficial for some aircraft configurations than others. This alternative approach means there is flexibility within the overall study to assess design decisions on the basis of the benefits they provide across many different concepts, rather than the effect for one specific concept.

Typically for this type of analysis there may be thousands of concepts that need to be analyzed individually. Each analysis is comprised of multiple calculations using analysis methods such as aerodynamics, mass estimation, mission modeling etc. The process is further complicated by discrete concept types, such as under wing or aft mounted engines or a series of options in the mission definition. Performing these analyses manually is not practical and so an automated approach is required.

Within IWATVP the proposed solution to these issues is the use of QinetiQ's RETIVO (Requirements Exploration, Technology Impact, and Value Optimisation) capability [3], which is described in more detail later. RETIVO is a software approach that allows the user to carry out assessments of concepts and technologies over a very broad design space. As outlined above, this flexibility is gained by adopting a relatively low level of aircraft geometry fidelity and associated performance modeling. RETIVO can be considered to model the design space in a broad but shallow approach. Favorable combinations of aircraft performance requirements and related technologies/systems choices can be explored using more detailed concept analysis and design capabilities. For example QinetiQ's complementary MDCAD (Multi-Disciplinary Concept Assessment and Design) capability [4], which is also described in more detail later, allows a more detailed concept geometry representation to be investigated and designed. Hence MDCAD can be considered as modeling the design space by a narrow but deep approach. This complementary use of RETIVO and MDCAD provides a systematic basis for both broad and deep studies, with the more detailed studies being used to underpin the broader, less detailed studies. In addition the more detailed MDCAD approach can be used to generate modeling information which can be used directly within broader RETIVO studies. Finally MDCAD can provide more detailed and specific data, such as detailed configuration geometry and structure, which can provide the starting point for the subsequent detailed design phase of an aircraft project.

\section{Broad Design Space Exploration (RETIVO)}

The general structure of RETIVO is shown in Figure 2 and is based around a flexible open software framework, that provides the data flow and structure into which individual tools or modules can be integrated, whether they simple 
equations, spreadsheets, program executables, or other bespoke or commercial analysis tools. This approach allows trusted modules, developed by individual disciplines for different purposes and without an original need to interface to other tools, to be integrated and re-used. This reduces the time and cost required to develop a RETIVO application suitable for a particular air vehicle project. Each module is "owned" and developed by a specialist discipline, such that the module is underpinned by a detailed understanding of the relevant subject which has been distilled into a rapid method that is suitable for this level of analysis. This ensures that the modules used in RETIVO, which fundamentally use quite a low level of modeling fidelity, still provide results which are trustworthy and which have been validated against higher fidelity sources of information.

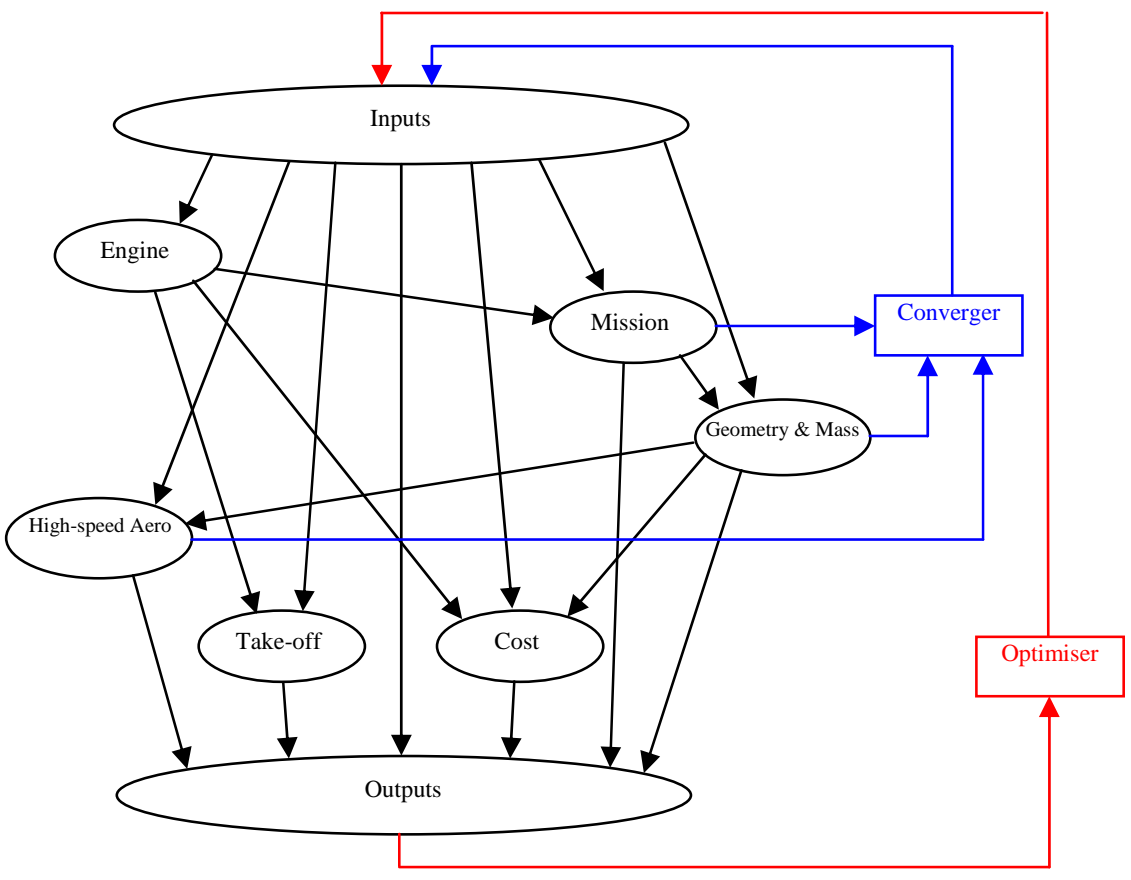

Figure 2. General structure of RETIVO

This approach also allows a RETIVO application to be tailored to a specific use; by careful choice of the most appropriate modules available, an appropriate trade off can be made between broad applicability and fidelity for the specific task at hand. For example, the focus within IWATVP is wing technologies, so a geometry and mass module that contains fuselage sizing and mass estimation techniques relevant to a conventional civil aircraft is appropriate. Generally, as the scope of a study is expanded many of the existing modules would still be appropriate, whilst some may require enhancement or replacement.

The flexibility in the RETIVO framework allows additional modeling capability to be added simply and quickly. All data output by one module is 
subsequently available to the other modules. Modules that have a loop dependency are iteratively converged via an inner 'converger'. For its use within IWATVP, this availability of data has allowed additional cost and emissions modules to be added to an original core of performance modules. The core modules allow the performance of the aircraft to be assessed with constraints upon performance attributes such as take-off distance, climb rates, and fuel consumption, whilst performing a defined mission. This core performance data is then used within the additional cost and emissions modules. The data produced by any module can be used as the focus for parametric trade studies, as objective and constraint functions as part of an optimisation process, or simply stored for information.

One of the most important factors in the choice or development of a module for use within RETIVO is that it should have a relatively wide range of applicability. One of the main benefits of RETIVO, compared to more specific concept design approaches, is that it avoids overly constraining the aircraft configuration modeling, allowing it to assess trends across a broad design space. The correct modeling of these trends across this broad design space is more important than absolute accuracy for a narrow set of configurations. For example, a study might be undertaken to consider how optimum wing area and engine thrust changes with decreasing aircraft mass. If the engine module was accurate for large engines, but this accuracy diminishes for smaller engines, then as the aircraft mass is reduced, and hence wing area and required thrust generally reduces, then the engine may be predicted to be heavier than it should be leading to misleading trends. An engine module which is comparatively less accurate, but which applies equally well to both large and small engines, will allow relative comparisons to be drawn.

This requirement for flexibility can be a major challenge to the specialists dealing with a given module, especially where traditional techniques rely on empirical methods. The wider range of applicability of a more physics based approach, such as computational fluid dynamics (CFD) or finite element analysis (FEA), is preferred, though this is not always possible, or practical, given the computational overhead associated with such modeling. Where a fully generalized physics-based approach is not available, it is essential to be aware of the limitations of each module and the constraints that this applies to the concepts being studied.

An important step in the application of RETIVO within the Integrated Wing project is to model the benefits and drawbacks of technology and system choices within RETIVO. At its simplest level this will take the form of "Technology A is likely to increase the wing weight by y\%, but bring a z\% saving in zero-lift drag". In this case, Technology A can be modeled within RETIVO by use of 'technology factors' applied to the wing weight and zero-lift drag calculated by the baseline modules. If it was required that an aircraft concept, employing Technology A, must achieve the same mission as an aircraft concept that does not incorporate this technology, then RETIVO may resize the concept to enable a like-for-like comparison. This ultimately means that the real impact of Technology A will extend far beyond merely wing weight and zero-lift drag. More complex technology or system choices may involve adding more detailed modeling within RETIVO in order to better represent the associated impact. For example, within Integrated Wing the geometry and mass module has been extended to model a wing box from first basic principles, in order to better capture the structural aspects 
of novel planform choices. This level of enhanced modeling is often not required or appropriate within RETIVO and hence the majority of technology and system choices will be captured through imposed technology factors.

\section{Detailed Concept Design (MDCAD)}

In order to investigate the impact of design decisions in greater detail than is available within a RETIVO type study, it is necessary to use a more detailed aircraft concept representation. In particular it may be necessary to adopt a more detailed representation of the geometry e.g. 3D configuration geometry, structural layout, packaging of systems, powerplant integration etc. Higher fidelity analysis methods would also be required to sufficiently resolve this additional level of geometry detail. Further, to achieve the realistic level of performance associated with these detailed concept models, it is necessary to ensure that a realistic level of design maturity is incorporated. This effectively means that it is necessary to design each concept, taking into account a wide range of factors, such as payload, range, take-off/landing performance, cruise conditions etc. As for the RETIVO approach, the implications of technology or system choices will then be added into these detailed concept models. Each concept will then be redesigned, in the light of these new design choices, in order to better assess the true associated benefits or penalties. Without this concept redesign step, the predicted impact would correspond to the addition of a technology or system as a post conceptual design step. Within Integrated Wing it is desired to assess how the original conceptual design would have changed, if the technology or system had been integrated from the outset. This enables the true value of a technology or system to be investigated.

To meet these requirements QinetiQ has established a Multi-Disciplinary Concept Assessment and Design (MDCAD) capability over many years [5-9]. Development of MDCAD has been driven both by the need to be applicable to novel aircraft configuration design, and to reduce the overall elapsed time for conceptual and preliminary design and performance assessment. The resulting capability uses computational physics based performance prediction tools where appropriate, such as Computational Fluid Dynamics (CFD) and Finite Element Analysis (FEA). Lower fidelity modules, such as those used in RETIVO, can also be used in combination where the particular application requirements allow. For example the mission modeling in RETIVO, which is relatively low fidelity, may also suffice for use in MDCAD. The addition of physics based performance prediction tools ensures that the resulting capability can be applicable to novel and conventional concepts. It also means that MDCAD can resolve more subtle effects due to design decisions than is possible in the less detailed models used in RETIVO. MDCAD makes extensive use of generalized Computer Aided Design (CAD) modeling, to provide a common aircraft geometry representation, which is central to the multi-disciplinary analysis and design process. The full capability is extensively automated to enable numerical optimisation driven design to be completed, in order to incorporate the required level of design maturity with reduced man effort. 
Within the Integrated Wing project QinetiQ is using MDCAD as a baseline environment, enabling the integration of further technology and system choices to be developed and studied relatively quickly.

MDCAD has been built upon high-fidelity computational physics based analysis and optimisation capabilities, much of which already existed within individual discipline groups within QinetiQ [10-13], to establish an integrated multi-disciplinary design optimisation capability. A critical factor necessary to achieve this has been the development of a common computer aided engineering (CAE) environment. This CAE environment consists of two main parts:

- Software framework for process automation and data exchange.

- Rules-based, parametric CAD model generator, which provides a multi-disciplinary, shared parametric representation of the configuration.

A bespoke framework utilizing Python based scripting has been developed and is used to automate the process. The rules-based, parametric CAD model generator is based upon the CATIA V5 commercial software product from Dassault Systèmes, enabling the automated generation of full external aircraft surfaces, structural layouts, local surface features (e.g. blending), deployable devices and internal packaging and systems. Computational physics analysis and optimisation tools are interfaced with this central CAD model within the software framework, to enable rapid analysis and optimisation. The exchange of information between the disciplines is standardized, for example the aerodynamics/structures exchange of loads and aero-elastic displacements. The baseline MDCAD framework used within the Integrated Wing project is shown schematically in Figure 3 for a generic civil aircraft case.

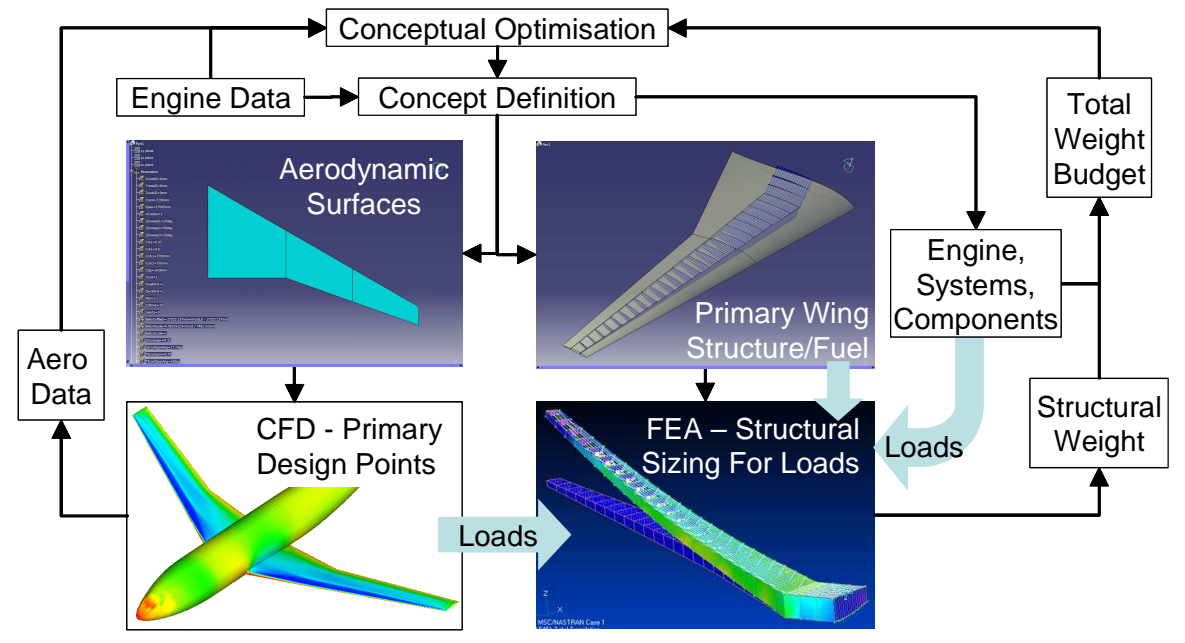

Figure 3. The Baseline MDCAD Framework

The aircraft concept is defined in terms of typical configuration parameters, such as wing planform and the fuselage length/diameter etc. Additionally, more detailed parameters are also specified which define the external aerodynamic 
surfaces e.g. camber, thickness and twist, and also the primary structural components e.g. spar and rib locations and sizing. Each of the configuration and detailed parameters is available for overall concept optimisation. These parameters are used to drive the CATIA V5 rules-based CAD geometry generator, which creates both the external CAD surfaces and the internal structural geometry.

Once the external surfaces have been generated it is then necessary to create the internal structural geometry. Using rules that define the location of the spars, rib and stringer spacing in addition to high-lift and control devices a structural CAD representation of the wing is generated. This CAD representation is used to calculate the capacity of the fuel within the wingbox region and is then translated into a FEA compatible model for use with MSc NASTRAN SOL200. Figure 4 shows the resultant structural geometries of four different planform variations. For these cases, the rules defining the spar location, rib spacing and number of stringers have remained constant although these can also be varied if required.

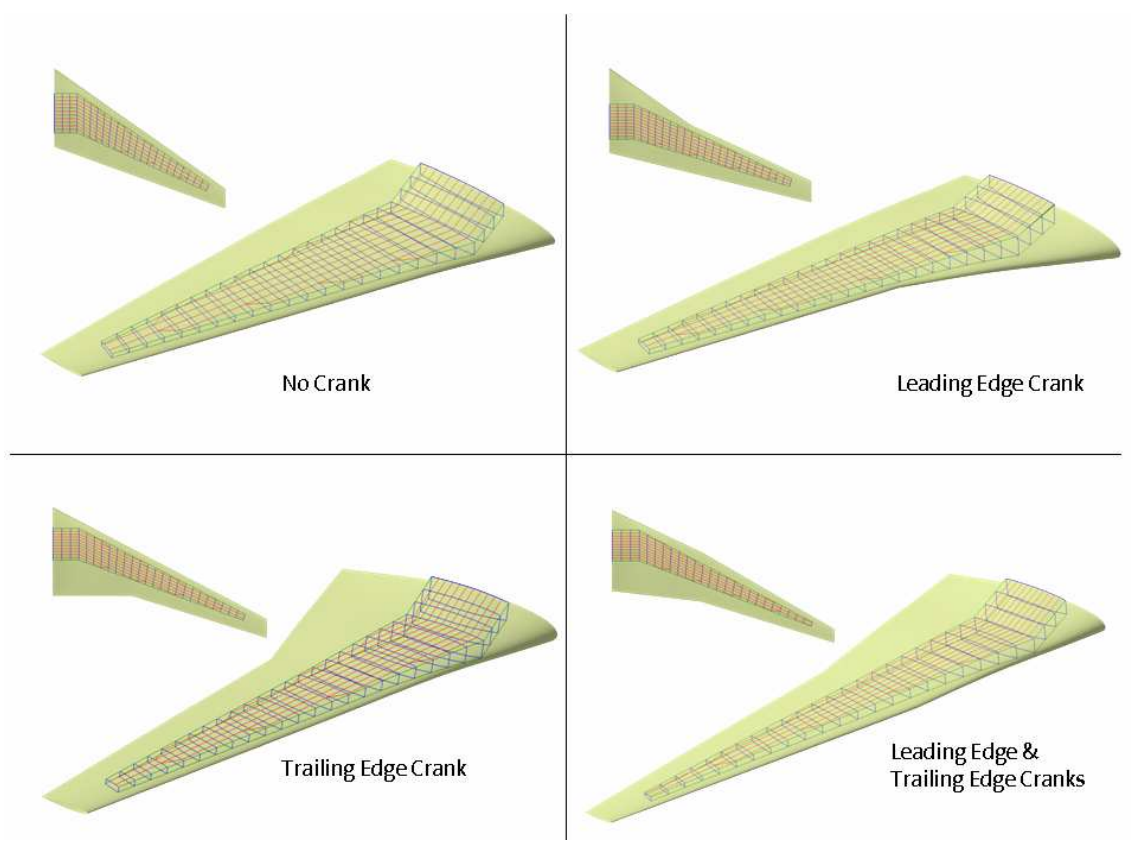

Figure 4. The structural layout for four different planforms

The external CAD surfaces are analyzed using CFD to generate aerodynamic performance information together with aerodynamic loading information. The primary structural components (spar, ribs, skin) are analyzed and their thicknesses optimized using FEA to generate structural weight information. During the structural analysis of the wing the aerodynamic loads are applied, together with loads associated with fuel carriage and other possible wing components e.g. engine installation, high-lift systems and landing gear.

The aerodynamic performance predictions from CFD, the weight predictions from FEA and other aircraft components, together with details of the chosen 
engine e.g. specific fuel consumption, are fed back into the overall optimisation problem formulation. A mission analysis module is used to calculate the performance of the aircraft from which various optimisation objective functions can be derived e.g. total fuel burn or maximum range. Typically numerous constraints are also included within the process e.g. operational requirements for fuselage cabin size, payload, cruise Mach number etc.

Each of the component parts of the overall process are set up as automated processes e.g. CAD generation, CFD grid generation, FEA model generation. The data exchange between these components can also be automated e.g. CAD input to CFD grid generation, transfer of aerodynamic loads into FEA. Hence the overall concept optimisation process can be fully automated and can be run without the requirement for user intervention.

The civil aircraft example described above highlights the importance of the central parametric CAD model within MDCAD. This CAD model provides a multi-level link between traditional conceptual design parameters (configuration definition) and preliminary design parameters (detailed features). The use of commercial CAD software within MDCAD also provides other benefits. For example the CAD software provides functionality for calculation of areas and volumes which can be used directly within constraints e.g. payload or fuel volume. Centres of gravity and inertias are also available and can be used as part of the structural model definition. Functionality for calculating distances between component CAD parts or features, or indeed identifying unwanted intersections between parts or features, can also be used within constraints.

The importance of a framework for linking the overall MDO process together is also clear from Figure 3. There is a requirement to establish an optimisation process across a network of machines. For example the CATIA V5 software is used on Windows platforms at QinetiQ, whilst the CFD and FEA software is usually run on a Linux multi-processor cluster. QinetiQ also uses the Python scripting to establish an overall optimisation process across a series of machines.

Within the MDCAD process the use of optimisation, and the associated higherfidelity analysis methods, to directly support concept analysis and design means that the conventional boundaries between conceptual design and preliminary design have been removed, and the two phases have to a large extent been merged. By using physics based analysis methods rather than simpler historical based correlations, the MDCAD approach provides generality to enable novel concepts to be assessed and designed, and improves the accuracy of the performance levels assumed during the conceptual design phase. This approach also results in the output of concepts which are more compatible with later stages of design helping to reduce overall design cycle.

The MDCAD process, presented in this paper, has been run to demonstrate the importance of optimizing both the aerodynamic and structural characteristics of a configuration simultaneously. For the cases investigated improvements in aircraft performance were noted through alterations to the planform, camber and thickness profiles resulting in changes to the external aerodynamic shape of the wing. These changes in external geometry and also due to the resultant change in the aerodynamic loading on the wing resulted in a new internal structural geometry. The thicknesses of the internal structural geometry were also optimized to 
minimize the overall wing weight. Constraints that were put on the configuration were satisfied including those dictating the performance of the aircraft ensuring that it was able to travel a given range, as well as meeting low speed performance and stability criteria.

Through the implementation of enhanced or additional modules within the process, the MDCAD capability can be used to further explore novel configurations, technologies or systems and the associated impact on the overall aircraft solution. Within the Integrated Wing Project enhanced versions of the MDCAD capability will be used to investigate the impact of several technology and system choices relevant to fuel systems, composites, landing gear and other systems.

\section{Conclusions}

The feasibility, conceptual design and preliminary design phases, when considered as part of one overarching activity, could be viewed as focussing upon an exploration of the 'achievable design space' of aircraft solutions and the resulting identification of the best solution to take forward into detailed design and production. In this context an 'aircraft solution' refers to matching the required performance and cost targets (customer requirements) with a viable aircraft concept which the manufacturer (and customer) is confident will achieve these targets. Ideally the priorities for particular performance and cost targets would be developed based upon an understanding of, and confidence in, these targets actually being achievable and representing some best design balance, such as between performance and cost. During these early phases of design there would hence a need to be able to trade-off requirements, to ensure concept viability and to be able to have confidence in the associated prediction of the anticipated final operational performance and cost for each aircraft concept.

To support this overarching design activity there would be a requirement for design capabilities which are fast, broadly applicable and sufficiently accurate. However these requirements conflict in practice presenting a dilemma for the development of such a design capability. In particular the requirement for a design approach to be both generally applicable and sufficiently accurate could potentially be addressed by solely using a relatively high fidelity modeling. However it is widely recognized that this high fidelity modeling is not always needed and can lead to undesirable complexity and a large increase in both manual and computational workload. Such an approach, if used in isolation, would inevitably lead to the possible space of design options being narrowed earlier than desired in order to reduce this workload. This situation would not represent an improvement compared to the traditional aerospace design process.

The current paper presents progress towards a more systematic approach for supporting the early phases of design, by using a combination of low and high fidelity tools, process automation and optimisation. The overall approach, as presented, incorporates two levels of design modeling, one for 'broad and shallow' exploration of the design space, the other enabling 'narrow and deep' investigations for more specific aircraft concepts. In practice these approaches are 
intentionally similar and ultimately many of the components can be common to both approaches. This potentially provides the basis for a single hybrid capability to be derived in the future, which would allow the modeling fidelity of different aspects of the design to be chosen according to the specific application requirements.

The design processes, which have been presented, use concurrent modeling of many different multi-disciplinary aspects of an aircraft and many associated measures of overall performance. This concurrent modeling ensures the value and viability of design decisions can be assessed in the context of the whole aircraft and the overall top level requirements.

There are several drivers for focusing on support for decision making in the early stages of design. Firstly there is a need to ensure that imposed top level requirements are viable and sensibly balanced. There is a desire to facilitate selection of the best aircraft solution which is matched to these requirements. Ultimately there may be an opportunity to de-risk the downstream design phases, by attempting to prevent possible problems from happening, through improved and higher fidelity upstream design.

\section{Acknowledgements}

The capabilities described have been funded over many years at QinetiQ and most recently through the Integrated Wing project, which is funded jointly by the UK Department for Business, Enterprise \& Regulatory Reform and QinetiQ. The authors would also like to show their gratitude to Airbus UK for the numerous discussions to date within the Integrated Wing project.

\section{References}

[1] ACARE, Strategic Research Agenda, Volumes 1 \& 2, 2002. Available at: http://www.acare4europe.org. Accessed on: May $1^{\text {st }} 2008$.

[2] Integrated Wing Aerospace Technology Validation Programme. Available at: https://www.integrated-wing.org.uk/default.htm. Accessed on: May $1^{\text {st }} 2008$.

[3] ELLSMORE PD, RESTRICK KE. Application of RETIVO to Civil Aircraft. Paper AIAA-2007-7808, Belfast, 2007.

[4] DOHERTY J, DEAN SRH. MDO-Based Concept Optimisation and The Impact of Technology and Systems Choices. Paper AIAA-2007-7806, 7th AIAA ATIO conference, Belfast, September 2007.

[5] FENWICK SV, HARRIS JapC. The application of Pareto frontier methods in the multidisciplinary wing design of a generic modern military delta aircraft. In proceedings of NATO RTO AVT symposium, Ottawa, October 1999.

[6] FENWICK SV, HARRIS JapC, DEAN SRH. Multi-disciplinary Optimisation to Assess the Impact of Cruise Speed on HSCT Performance. Paper AIAA-2005-4538, Albany, 2004.

[7] BARTHOLOMEW P. Structural Optimisation within the Multidisciplinary Design Process. In proceedings of 5th ASMO UK/ISSMO conference on Engineering Design Optimisation, Stratford-upon-Avon, July, 2004. 
[8] DOHERTY JJ. Rapid Multi-Disciplinary Analysis and Optimisation of Novel Air Vehicle Configurations. In proceedings of CEAS/KATnet Conference on Key Aerodynamic Technologies, Bremen, 2005.

[9] DOHERTY JJ, DEAN SRH. The Role of Design Optimisation within the Overall Vehicle Design Process. In proceedings of 6th ASMO UK / ISSMO conference on Engineering Design Optimization, Oxford, July 2006.

[10] BARTHOLOMEW P, VINSON S. STARS: Mathematical Foundations; In Software Systems for Structural Optimisation. Birkhauser Verlag, Basel 1993.

[11] DOHERTY JJ, PARKER NT. Dual Point Design of a Supersonic Transport Wing using a Constrained Optimisation Method. In proceedings of 7th European Aerospace Conference - The Supersonic Transport of Second Generation, Toulouse, 1994.

[12] ROLSTON SC, DOHERTY JJ, EVANS TP, GRENON R, AVERARDO MA. Constrained Aerodynamic Optimisation of a Supersonic Transport Wing: a European Collaborative Study. Paper AIAA-98-2516, Albuquerque, June 1998.

[13] HACKETT KC, REES PH, CHU JK. Aerodynamic Design Optimisation Applied to Civil Transports with Underwing Mounted Engines. In proceedings of ICAS Conference, Melbourne, 1998. 